\title{
Proximal buddy in jail technique: A bail out technique to increase guide support
}

\author{
Tao Tu, Jianjun Tang, Zhenfei Fang, Xinqun Hu, Liang Tang, \\ Yanshu Zhao, Qiming Liu, Shenghua Zhou \\ Department of Cardiovascular Medicine, The Second Xiangya Hospital, \\ Central South University, Changsha, Hunan, PR China
}

\begin{abstract}
Background: During percutaneous coronary intervention, "buddy-in-jail" technique is often used to facilitate stent delivery in complex coronary artery lesions. However, the safety and efficacy of this technique when used with different jailed wire and applied in different target vessel lesions remain elusive. The aim of this retrospective study was to analyze the effectiveness of "buddy-in-jail" technique in the tortuous and rigid lesions of both the common and neighboring coronary arteries. The effectiveness between hydrophilic-coated and non-hydrophilic-coated guide wire as jailed wires was also compared. Methods: The "buddy-in-jail" technique was applied in 15 patients after failed balloon or stent delivery into the target vessel lesion from June 2014 to December 2016. The safety and effectiveness of the "bud$d y$-in-jail" technique was compared in the tortuous and rigid lesions of both the common and neighboring coronary arteries and between hydrophilic-coated and non-hydrophilic-coated "jailed" wires.

Results: Stent delivery was successful in 13 (86.7\%) patients with the use of "buddy-in-jail" technique. The success rate was similar to the group using the common artery (87.5\%) as a "buddy" vessel and the group using a neighboring artery (85.7\%) as a "buddy" vessel ( $p>0.05)$, and between hydrophilic-coated (100\%) and non-hydrophilic-coated "jailed" wire (77.8\%) group $(p>0.05)$. All wires were successfully extracted without complications.

Conclusions: The "buddy-in-jail" technique offers a potential alternative approach for the distal stent delivery in both the common and neighboring coronary arteries. Also, both hydrophilic and non-hydrophilic-coated wire could be safely and effectively used as "jailed" wire. (Cardiol J 2018; 25, 2: 159-164)

Key words: "buddy-in-jail” technique, distal stent delivery, hydrophilic-coated guide wire, complex coronary artery lesions
\end{abstract}

\section{Introduction}

The equipment and techniques for percutaneous coronary artery intervention (PCI) have been considerably improved over recent decades, and a majority of coronary lesions could be successfully treated by PCI. However, it remains technically challenging for stenting severely and diffusely diseased coronary arteries, such as vessels with proximal vessel tortuosity, rigid segments due to calcified atherosclerotic plaques or previously stented segments [1-3]. One of the biggest challenges for interventional cardiologists is to handle the "balloon uncrossable" lesions, multiple techniques including the use of stiffer guide wires, buddy wires, more aggressive guide catheter support, guide catheter extensions, use of anchor balloons, and proximal occlusion balloons are proven to be useful in these cases to achieve success in the final procedure $[2,3]$.

The "buddy wiring" technique serves as an effectively and routinely used option to facilitate

Address for correspondence: Jianjun Tang, MD, PhD, Department of Cardiovascular Medicine, The Second Xiangya Hospital, Central South University, NO.139 Renmin Road, Changsha, Hunan, 410011, PR China, tel: +86 73185292012 , fax: +8673185533525, e-mail: tom200210@sina.com 
the delivery of balloons and stents through tortuous coronary vessels. During the classical process, a second or "buddy" wire should be placed parallel to the wire in order to guide catheter-balloons and stents. Finally, the "buddy" wire will be removed after the stent is positioned in the target lesion [4]. The modified "buddy" wire technique in which the buddy wire was "jailed" by stenting a proximal lesion has been reported in several cases and named as "buddy-in-jail" technique $[4,5]$. This technique facilitates the balloon(s) and stent(s) over the nonbuddy and non-jailed wire and makes the treatment of distal lesions easier $[4,5]$.

In this retrospective study, the validity of "buddy-in-jail" technique was investigated in both the common and neighboring coronary artery and compared this validity in hydrophilic-coated and non-hydrophilic-coated guide wire.

\section{Methods}

The patient population consisted of consecutive patients who were hospitalized in our department and received PCI for treatment of coronary artery disease between 2014 and 2016. Owing to the failure of standard methods in achieving stent delivery (such as supportive guide catheters, "mother-in-child catheter", buddy wire, "anchoring” balloons, distal balloon deflation), "buddy-injail" technique was performed to increase support for stent delivery. According to the jailed wire, the patients were divided into the hydrophilic-coated guide wire group and non-hydrophilic-coated guide wire group.

\section{The buddy-in-jail technique}

All patients choose transradial approach. As shown in Figure 1, stent implantation was planned on both proximal and distal diseased segments of the right coronary artery (RCA). However, the standard supportive techniques, such as supportive guide catheters [6], catheters with extra support shape [6], "mother-in-child catheter" [7], GuideLiner [8, 9] or deep seating $[10,11]$, buddy wiring, anchor wiring and anchor ballooning failed to deliver a stent or balloon to the distal diseased segment after the guide wire was advanced to the distal diseased segment.

Then, the proximal vessel as the "jailed" position was stented to jail the buddy wire when a second hydrophilic/non-hydrophilic-coated buddy wire (jailed wire) was delivered to the distal vessel. At this time, benefited from the additional support offered by the wire being fixed by proximal stent deployment, the guiding catheter-stent-jailed wire

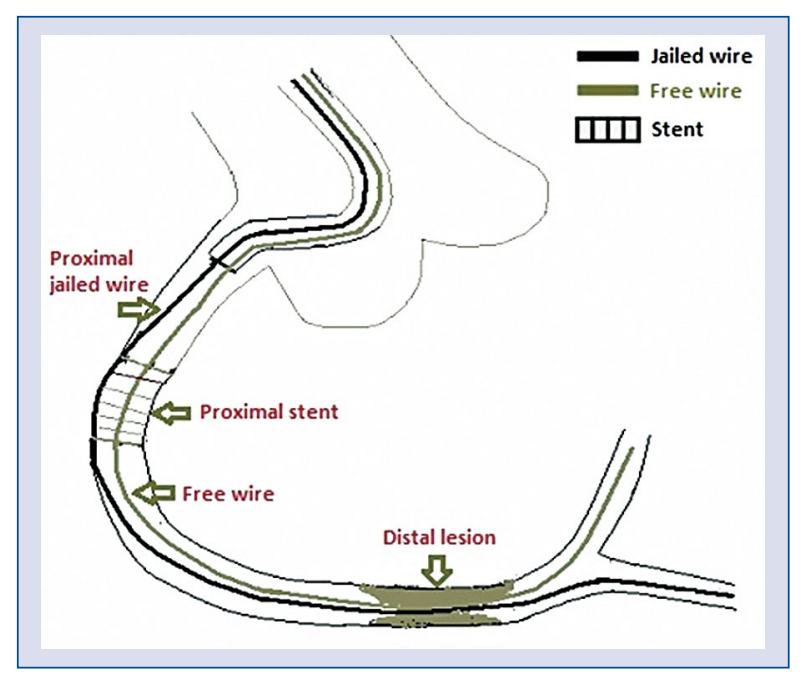

Figure 1. The buddy-in-jail technique for right coronary artery.

could form a new and powerful support system to overcome vessel resistance and facilitate delivery of a stent or balloon to the distal lesion. Before deployment of the distal stent, the jailed buddy wire should be removed to avoid "double jailing". In the present study, the jailed position vessel could be the common artery (Fig. 2A) or the neighboring artery (Fig. 2B): the left anterior descending (LAD) lesion is the jailed position and the left circumflex (LCX) lesion is the second stent position.

There are three notable points which should not be missed during the procedure. First, though the pressure (12-14 atm) used in this study was safe to release the proximal stent to jail the buddy wire, high pressure ( $>14 \mathrm{~atm}$ ) should be used very carefully to avoid wire entrapment or fracture. Second, as shown in Figure 2B, a second wire should be positioned in the stent before removing the jailed wire in the LAD to avoid fatal risks brought by stent dislocation. Third, after removing the jailed wire, a high-pressure post dilatation should be performed to avoid malposition after modest pressure deployment of the first stent.

The study was approved by the local bioethical committee and all patients gave their informed consent.

\section{Statistical analysis}

Continuous variables are presented as mean \pm standard deviation, and categorical variables are expressed as numbers (percentages). Data comparison between the two groups was performed via Student $t$ test for continuous variables or Fisher 


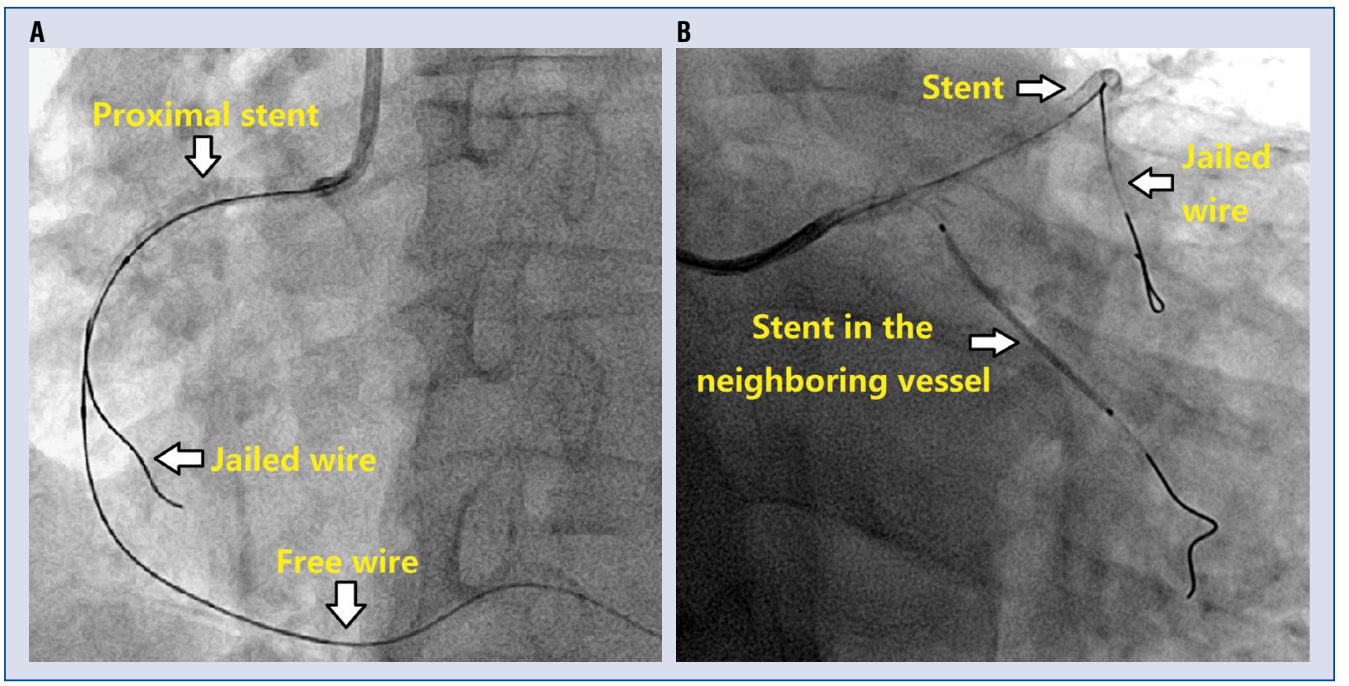

Figure 2. The buddy-in-jail technique were used in the common/neighboring vessel. $\mathbf{A}$. The proximal lesion in the common vessel was chosen as jailed position; B. The lesion in left anterior descending was chosen as the jailed position to increase the support for the left circumflex lesion stent.

Table 1. The clinical and procedural characteristics.

\begin{tabular}{lc}
\hline Characteristics & Number of patients \\
\hline Age [years] & $65.4 \pm 8.6$ \\
Sex (male/female) & $8 / 7$ \\
Disease artery (RCA/LAD/LCX) & $6 / 3 / 6$ \\
Lesion characteristics: & \\
Severe calcification & 5 \\
Chronic total occlusion & 4 \\
Tortuosity & 3 \\
Calcification combine with angulation & 1 \\
Calcification combine with tortuosity & 2 \\
Jailed vessels (common/neighboring) & $8 / 7$ \\
Guide wire (hydrophilic/non-hydrophilic-coated) & $6 / 9$ \\
Deployment pressure of jailed position [atmospheres] & $13.6 \pm 1.2$ \\
Length of the stents used for the trapping [mm]: 14/18/20/21/24/28/33 mm & $2 / 4 / 2 / 3 / 2 / 1 / 1$ \\
\hline
\end{tabular}

LAD — left anterior descending artery; LCX — left circumflex artery; RCA — right coronary artery

exact test for categorical variables. Statistical significance was established as $\mathrm{p}<0.05$. All statistical analysis was performed using SPSS Statistics version 19.0 (IBM Corp., Armonk, New York).

\section{Results}

\section{Patient characteristics}

The "buddy-in-jail" technique was applied in 15 patients who had appropriate "jailed" diseased segment and the standard supportive techniques failed to deliver a stent or balloon to the distal diseased segment. The average age was $65.4 \pm$ \pm 8.6 years in this patient cohort. The "buddy-in-jail" technique was applied more often in RCA and LCX lesions than in LAD lesion. Distortion and calcification was the major cause responsible for the failure of stent delivery with standard supportive techniques. Patient and coronary lesion characteristics are presented in Table 1.

\section{Validity of different jailed vessels and wires}

During the PCI, stent delivery to the distal lesion was successful in 13 (86.7\%) patients with 
Table 2. The clinical characteristics between hydrophilic and non-hydrophilic-coated jailed wire patients.

\begin{tabular}{lccc}
\hline & Hydrophilic-coated wire & Non-hydrophilic-coated wire & P \\
\hline Age [years] & $67.2 \pm 8.6$ & $64.2 \pm 8.9$ & 0.533 \\
Sex (male/female) & $4 / 2$ & $5 / 4$ & 0.545 \\
Jailed vessel (common/neighboring) & $3 / 3$ & $5 / 4$ & 0.622 \\
Success rate & $100 \%(6 / 6)$ & $77.8 \%(7 / 9)$ & 0.486 \\
\hline
\end{tabular}

the application of "buddy-in-jail" technique. There was no significant difference between the group using the common artery $(87.5 \%, 7 / 8)$ and the group using the neighboring artery $(85.7 \%, 6 / 7)$ as the "buddy" vessel ( $\mathrm{p}>0.05)$. However, stent delivery to the distal lesion was not successful in 2 patients with chronic total occlusion despite the use of "buddy-in-jail" technique. After the guide wire passed the distal lesion, coronary rotational atherectomy was performed in these 2 patients. Finally, stent delivery was successful in 1 patient but failed in another patient.

There were no differences in age, sex and jailed vessel between the hydrophilic and nonhydrophilic-coated buddy wire groups. The success rate of hydrophilic-coated wire group (100\%) and the non-hydrophilic-coated wire group (85.7\%) was also similar $(\mathrm{p}=0.486)$ (Table 2$)$. During PCI, all the jailed buddy wires were removed smoothly and there were no serious complications related to this procedure.

\section{Discussion}

The main finding of the present study is that the "buddy-in-jail" technique could offer supplementary support for distal stent delivery in both common and neighboring coronary arteries. Also, both hydrophilic and non-hydrophilic-coated wire can be safely and effectively used as "jailed" wire.

However, considering the potential risk of failure of "buddy-in-jail" technique, we should first seek answers from available options during stenting difficult lesions before seeking help from the jailed wire. First, a guiding catheter with good backup force is very important to facilitate the delivery of stents into complex coronary lesions. The size and shape of the guiding catheter are two important factors contributing to the generation of backup force [6]. It is known that a 7 Fr size guiding catheter can generate stronger backup force than a smaller guiding catheter, the backup force of the Amplatz $\mathrm{L}$ is greater than that of Judkins $\mathrm{R}$ in right coronary artery interventions [6]. Mother-in-child catheter technique is quite an effective way to deliver stents into complex coronary lesions. A 4 Fr or 5 Fr size into 6 Fr or 7 Fr size guiding catheter could be chosen to enhance the backup force of guiding catheter. This technique showed success of stent delivery in $>90 \%$ of cases in which the conventional technique had failed [7]. A recent study showed that GuideLiner motherand-child guide catheter extension, as a simple adjunctive tool and rapid exchange guide catheter extension system, facilitated the mother-in-child technique and was successfully used in patients with complex lesions [8, 9]. Deep seating is a supplementary method for increasing the support of standard guiding catheter and mother-in-child catheter technique $[10,11]$. This technique is feasible and safe, and may markedly increase the success rate of stent delivery in a very complex coronary anatomy and when standard guiding catheters have failed $[10,11]$. Second, guiding wire techniques such as stiff wire, buddy wiring and anchor wiring play other important rolesin facilitating the delivery of stents into complex coronary lesions. The buddy wire technique is the most often applied technique during stenting and it uses a second wire forwarded into the distal of the target vessel alongside the main one. Sometimes there are two buddy wires and those buddy wires could act as a track and help the progression of the balloon or the stent delivery to the target lesion. Then, the buddy wire should be removed as soon as the undeployed stent is located in the target lesion [4, 12]. The anchor wire technique is a little bit different from buddy wire. When deliverability is limited during PCI, a second guide wire is inserted into a nontarget artery. Then, another attempt is made to deliver the device to the target lesion [13]. These techniques could all improve the support of guide catheter system and device deliverability during PCI. Third, anchor ballooning belongs to the key balloon technique to increase the backup support of guiding catheter after optimal lesion preparation with compliant or non-compliant balloon. This technique is based on the buddy wire and anchor 
wire technique. To get superior guiding catheter support, it anchors the guiding catheter with another balloon inflated in a non-target vessel or distal bed of the target vessel, which is proven to be helpful for a balloon and stent to cross the target lesion [14]. The anchor balloon should use only low pressure (6-8 atmospheres or less) to minimize the risk of branch vessel tear. Other techniques, such as deep inspiration may also facilitate difficult stent delivery in the native coronary arteries [15].

In the overwhelming majority of cases, the above standard support techniques could be sufficient in delivering the stent into complex lesions such as calcified, bent, or diffuse lesions. However, few of the extremely complex cases, stent delivery requires additional technical solutions. Asfor those cases, supplementary support offered by jailed wire could be useful in those multiple lesion target vessels.

In the present study, among the 15 patients who have "jailed" diseased lesions and complex coronary lesions, successful stent delivery was achieved in 13 patients with the help of "buddy-in-jail” technique. RCA and LCX lesions were related to more often to stent delivery difficulties, especially when a diffuse lesion was combined with distortion and calcification. In these patients, standard methods like supportive guide catheters, "motherin-child catheter", catheters with extra support shape or deep seating, buddy wire, "anchoring" balloons were used and failed. As described in some case reports, "buddy-in-jail" technique is an inexpensive and effective method to secure the guiding catheter and could provide better support making treatment of distal lesions easier $[5,16]$. Later, Dangoisse et al. [4] promoted the distal "buddy-in-jail" technique to 10 patients after the first successful stent delivery into a calcified and tortuous large RCA. Thus, the additional support from the jailed buddy wire beneath could provide enough anchorage force for the guiding catheterstent-jailed wire support system to facilitate stent delivery in difficult lesion situations. However, the clinical use of this technique is limited when there is additional proximal disease where stenting in the common artery is needed. In the present study, the proximal "buddy-in-jail" technique was not only promoted in 8 patients in the common coronary artery, but also extended this technique to the neighboring coronary artery when there was an additional vessel lesion requiring stent implantation in the nearby vessel. Meanwhile, similar success rate was achieved from the common artery group and neighboring artery group. Therefore, both proximal and distal vessel lesions could be the jailed position, and the additional support from the jailed buddy wire could be shared and used in the whole guiding catheter-stent-jailed wire support system, even for the stent delivery in the vessel lesion of a neighboring artery.

During the PCI of the branch disease, the protect wire buried in the branch may bring new problems during withdrawal. The "jailed" wire could be hard to draw out or fractured in the removing process, especially when there is a diffuse calcified lesion [17]. Thus, cardiologists would most likely choose hydrophilic-coated buddy wire to protect the branch vessel and reduce the potential risk related to the above conditions. However, the coating may exfoliate or damage when drawing out the hydrophilic-coated wire. Actually, coated wires are more resistant to retrieval damage and more efficient in crossing the side branch ostium than non-coated wires [18]. In the present study, both hydrophilic and non-hydrophilic-coated jailed wire could provide enough additional support and were removed smoothly and no serious complication occurred. The success rate between two groups was also similar.

Percutaneous coronary intervention of tortuous saphenous vein grafts can be difficult during balloon or stent advancement. Because of the specificity and fragility of saphenous vein grafts, few complex PCI techniques have been performed on tortuous saphenous vein grafts lesion. Movahed [19] reported a reverse "Movahed Maneuver" during vein graft interventions which was successful in many difficult vein graft intervention cases due to tortuosity. There is, however, no report describing the application of buddy in jail technique in any vein graft interventions. Considering the potential risk of vein graft dissection and fracture, we do not suggest the use of this technique in PCI of vein grafts.

Though the risks of using this "buddy-in-jail" technique are seldom reported, we should also pay attention to the potential risks of the whole procedure, especially the procedure of pulling back the distally jailed wire. High pressure on the deployment of jailed stent may trap the wire between a stent and calcified tissue and result in difficulties in pulling back the distally jailed wire, which may lead to wire entrapment and/or fracture, even stent dislocation $[4,5]$. The whole procedure should be performed gently and slowly. Violent action on pulling back the jailed wire may increase risk of coronary artery opening dissection and proximal stent dislocation due to catheter fixation and deep seating $[4,5]$. We must try to avoid the situation of 
both wires jailed or jailing the same wire with two consecutive stents. As soon as the proximal stent is well positioned and before its release, the wire that is not loaded with the stent must be pulled back $[4,5]$. People might be concerned about the possible peeling of the hydrophilic coating during the pull-back procedure, but this phenomenon was not observed in our patient cohort.

\section{Limitations of the study}

The main limitation of the present study is the small number of patients, due to the lower prevalence rate of "balloon uncrossable" lesions. Due to the small patient number in each group, differences in clinical characteristics between hydrophilic and non-hydrophilic-coated jailed wire groups may have been "overlooked". Meanwhile, the "buddy-in-jail technique" should be used as a complementary option for stent delivery as the deployment of the first proximal stent may hamper the delivery of the second stent to the distal vessel. If the technique had failed, there would be almost no chance to perform distal rotablation over the proximal implanted stent. Additionally further large-scale and prospective studies are warranted to validate the results derived from this retrospective study.

\section{Conclusions}

The "buddy-in-jail" technique offers a potential alternative approach for the distal stent delivery in both the common and neighboring coronary artery. Also, both hydrophilic and non-hydrophiliccoated wire could be safely and effectively used as "jailed" wire.

\section{Conflicts of interest: None declared}

\section{References}

1. Lee MS, Park KW, Shlofmitz E, et al. Comparison of Rotational Atherectomy Versus Orbital Atherectomy for the Treatment of Heavily Calcified Coronary Plaques. Am J Cardiol. 2017; 119(9): 1320-1323, doi: 10.1016/j.amjcard.2017.01.025, indexed in Pubmed: 28258729.

2. Karacsonyi J, Karmpaliotis D, Alaswad K, et al. Prevalence, indications and management of balloon uncrossable chronic total occlusions: Insights from a contemporary multicenter US registry. Catheter Cardiovasc Interv. 2017; 90(1): 12-20, doi: 10.1002/ ccd.26780, indexed in Pubmed: 27650935.

3. Tomey MI, Sharma SK. Interventional options for coronary artery calcification. Curr Cardiol Rep. 2016; 18(2): 12, doi: 10.1007/ s11886-015-0691-8, indexed in Pubmed: 26768738.

4. Dangoisse V, Guédès A, Schroëder E. Distal 'buddy-in-jail' technique: a complementary 'Jail with stent' method for stent deliv- ery. Acute Card Care. 2014; 16(1): 28-33, doi: 10.3109/17482941. 2013.869342, indexed in Pubmed: 24552227.

5. Bagnall AJ, Spratt JC. The "buddy-in-jail" technique: a novel method for increasing support during percutaneous coronary intervention. Catheter Cardiovasc Interv. 2009; 74(4): 564-568, doi: 10.1002/ccd.22049, indexed in Pubmed: 19472348.

6. Ikari Y, Masuda N, Matsukage T, et al. Backup force of guiding catheters for the right coronary artery in transfemoral and transradial interventions. J Invasive Cardiol. 2009; 21(11): 570-574, indexed in Pubmed: 19901410.

7. Uchida Y, Nakashima H, Takeshita S. Modified mother-child technique using a buddy wire. J Invasive Cardiol. 2014; 26(5): E52-E53, indexed in Pubmed: 24791725.

8. Sharma D, Shah A, Osten M, et al. Efficacy and safety of the guideliner mother-in-child guide catheter extension in percutaneous coronary intervention. J Interv Cardiol. 2017; 30(1): 46-55, doi: 10.1111/joic.12354, indexed in Pubmed: 27862338.

9. Brown BJ, Abbott JD. A helping hand: GuideLiner use to facilitate stent delivery. Catheter Cardiovasc Interv. 2016; 88(7): 1065-1066, doi:10.1002/ccd.26866, indexed in Pubmed: 27976540 .

10. Bartorelli AL, Lavarra F, Trabattoni D, et al. Successful stent delivery with deep seating of 6 French guiding catheters in difficult coronary anatomy. Catheter Cardiovasc Interv. 1999; 48(3): 279-284, indexed in Pubmed: 10525228.

11. Hoshino Y, Kunii H, Misaka T, et al. Deep seating of 5 Fr. guiding catheter across the stenosis with 5 in 7 method was effective for severely calcified lesion. Cardiovasc Interv Ther. 2013; 28(1): 87-90, doi: 10.1007/s12928-012-0119-y, indexed in Pubmed: 22930372.

12. Burzotta F, Trani C, Mazzari MA, et al. Use of a second buddy wire during percutaneous coronary interventions: a simple solution for some challenging situations. J Invasive Cardiol. 2005; 17(3): 171-174, indexed in Pubmed: 15867450.

13. Hamood H, Makhoul N, Grenadir E, et al. Anchor wire technique improves device deliverability during PCI of CTOs and other complex subsets. Acute Card Care. 2006; 8(3): 139-142 , doi: $10.1080 / 17482940600885469$, indexed in Pubmed: 17012127.

14. Takahashi S, Saito S, Tanaka S, et al. New method to increase a backup support of a 6 French guiding coronary catheter. Catheter Cardiovasc Interv. 2004; 63(4): 452-456, doi: 10.1002/ccd.20223, indexed in Pubmed: 15558766.

15. Attaran RR, Butman S, Movahed MR. Going around the bend: deep inspiration facilitates difficult stent delivery in the native coronary arteries. Tex Heart Inst J. 2011; 38(3): 270-274, indexed in Pubmed: 21720470.

16. Kaluski E, Tsai S, Milo-Cotter O. Buried wire technique: enhancing support method for complex percutaneous interventions and stenting. J Invasive Cardiol. 2007; 19(4): 195-196, indexed in Pubmed: 17404407.

17. Balbi M, Bezante GP, Brunelli C, et al. Guide wire fracture during percutaneous transluminal coronary angioplasty: possible causes and management. Interact Cardiovasc Thorac Surg. 2010; 10(6): 992-994, doi: 10.1510/icvts.2009.227678, indexed in Pubmed: 20351016.

18. Pan M, Ojeda S, Villanueva E, et al. Structural damage of jailed guidewire during the treatment of coronary bifurcation lesions: a microscopic randomized trial. JACC Cardiovasc Interv. 2016; 9(18): 1917-1924, doi: 10.1016/j.jcin.2016.06.030, indexed in Pubmed: 27659568.

19. Movahed MR. Reverse movahed maneuver: maximal expiration maneuver during saphenous vein graft intervention for facilitated balloon and stent delivery in tortuous vein grafts a novel technique. Int J Angiol. 2016; 25(5): e66-e69, doi: 10.1055/ s-0034-1395977, indexed in Pubmed: 28031658. 